\title{
Experimental study of multiphase flow in a model gearbox
}

\author{
V. Chernoray ${ }^{1} \&$ M. Jahanmiri ${ }^{1,2}$ \\ ${ }^{1}$ Division of Fluid Dynamics, Department of Applied Mechanics, \\ Chalmers University of Technology, Göteborg, Sweden \\ ${ }^{2}$ Department of Mechanical and Aerospace Engineering, \\ Shiraz University of Technology, Iran
}

\begin{abstract}
This study concerns dynamics of a two-phase flow around a rotating solid body. Under consideration is a model of a gear wheel in a gearbox which rotates and is partially submerged in oil. The flow of interest is complex and involves effects of free surface dynamics, rotation, and formation of bubbles and drops. Occurring flow regimes include laminar, transitional and turbulent. The major focus of the investigation is on details of the developed flow, and the purpose is validation of numerical methods developed for design and optimization of such components. Current experiments are performed in a test rig which is modelling a generic simplified gearbox with a single isolated rotating wheel. The flow measurements are carried out by using particle image velocimetry (PIV) and the test rig is specially designed for this purpose with the optical access maximized. The flow similarity with respect to a real gearbox is fully maintained and the working fluid is a transparent mineral oil. The PIV measurements are performed at four different rotation speeds for two different wheel configurations in order to cover a spectrum of operational conditions needed for numerical modelling. The emphasis is on the result of experiments on a smooth wheel. The measurements are providing velocity distribution around the wheel and details on bubble and drop distribution.
\end{abstract}

Keywords: bubble and drop dynamics, rotating wheel, splash, surface tension, gear box, PIV measurements. 


\section{Introduction}

Multiphase flows are present in numerous engineering applications. For better development of applied methods used in prediction and optimization of engineering flows a deep understanding of underlying flow phenomena is crucial. Nevertheless it is known that obtaining knowledge on complex multiphase flows occurring in industrial processes [1] is often a challenging task where both numerical simulations and experimental investigations can be very demanding.

Gear lubrication is a significant concern in a wide range of industries which use a power transmission. SAAB Powertrain Sweden is developing computational fluid dynamics (CFD) models for predicting and optimizing the oil flow around rotating components in gearboxes. The main objective is to optimise the losses in a gearbox and thereby decrease the fuel consumption and exhaust emissions of vehicles. Furthermore, an effective lubrication reduces the operating cost of a gearbox not only by improving the efficiency of transmissions but also by prolonging the component lifetime. Increasing the efficiency of gearboxes has a great market potential not only in the vehicle industry but also in many other industries where the power transmission is used such as the pulp industry, mining industry, wind and hydropower industry.

The gearbox optimization is rather complex problem since in a gearbox the oil is used not only to reduce the friction between the gearwheels but also acting as a cooling fluid. This means that both the oil amount and the oil flow have to be optimised. The numerical prediction of the oil flow in a gearbox is not a trivial task and reliable measurements for validation of CFD models are required.

There are surprisingly few studies which are focussing on the actual details of the gearbox flow. The oil and airflow related losses are contributing to the socalled load-independent spin power losses which include the air windage, oil churning, inertial power loss from an impinging oil jet (for the case of jet lubrication), fluid trapping and squeezing between meshing gear teeth, rotating seals, and bearings [2]. Further discussion on importance of these losses can be found in several recent works devoted to the gearbox efficiency [3-5]. A particular insight into the fluid mechanics of gearboxes is attempted only in a fewer works. In papers [6-8] the studies were performed by using CFD and in papers $[9,10]$ the CFD was used in combination with experiments. However the experimental data available in these works are very limited.

Current experimental study was initiated in order to complement the above research. The investigation is performed by using PIV and the flow similarity with respect to a real gearbox is maintained.

\section{Experimental set-up}

The gearbox model with single rotating wheel is shown in Fig. 1. The test rig is representing a simplified model of a gearbox. The major idea is to isolate the splash effects from the wall effects and for this purpose the box size is enlarged as compared to the real gearbox. The rig is specially designed for using PIV 
which means that the optical access to the flow is maximized. The dimensions of the box are $0.82 \times 0.52 \times 0.32 \mathrm{~m}^{3}$. The gear wheel is made interchangeable and the wheel rotation speed can be varied to investigate different operational conditions. The wheel models are produced by stereo lithography method and used wheels have diameter of $0.22 \mathrm{~m}$ and thickness of $28 \mathrm{~mm}$. Two wheel configurations are used, a flat wheel, which can be seen in figure, and a spur gear wheel with 67 teeth.

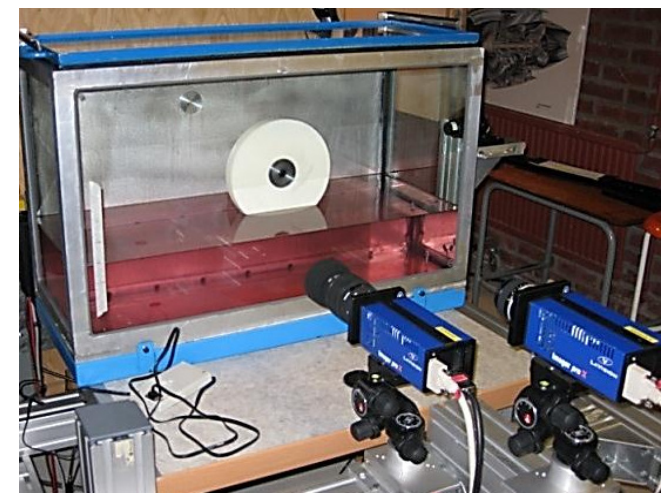

Figure 1: Model gearbox and gear wheel. showing the experimental configuration and position of the CCD cameras.

Two-dimensional, two-component PIV measurements (2D-2C) are reported in this paper. The laser plane was positioned at wheel symmetry plane (midpoint of the wheel thickness) and perpendicular to the axis of rotation. The camera fields of view are depicted schematically in Fig. 2. Each of two cameras was adjusted for own field of view for easiness of obtaining repeatable experiments.

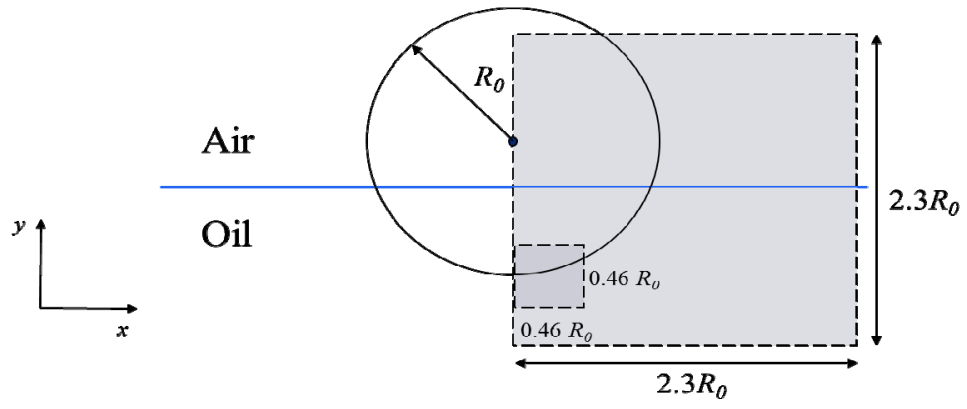

Figure 2: Camera fields of view in relation to the wheel.small FOV is $50 \times 50 \mathrm{~mm}^{2}$ and large FOV is $250 \times 250 \mathrm{~mm}^{2}$.

The optical axis of camera is normal to the plane of the light sheet. The camera fields of view are $50 \times 50 \mathrm{~mm}^{2}$ and $250 \times 250 \mathrm{~mm}^{2}$. To obtain the flow information around the other half of the wheel the wheel rotation direction was 
changed. Due to the flow symmetry this was equivalent to moving the field of view to other side of the wheel.

The CCD cameras have resolution of $2048 \times 2048$ pixel (LaVision Imager pro $\mathrm{X} 4 \mathrm{M})$ with $7.4 \times 7.4 \mu \mathrm{m} 2$ pixel size. The laser is pulsed dual-cavity Nd-YAG laser with $400 \mathrm{~mJ} /$ pulse at $532 \mathrm{~nm}$ (Spectra-Physics). The thickness of the light sheet was approximately $5 \mathrm{~mm}$. The flow was seeded with fluorescent particles (PMMA Rhodamine B) with diameter of $1-20 \mu \mathrm{m}$ and density of $1.5 \mathrm{~kg} / \mathrm{m}^{3}$. The fluorescent light from particles is separated by a low-pass filter.

The working fluid is a transparent synthetic oil which has same viscosity at room temperature as gearbox oil at design temperature. In this way the Reynolds number similarity with a real gearbox is maintained.

\section{Results and discussion}

\subsection{Oil splash visualizations}

The complexity of the flow in the model gearbox can be understood from visualizations presented in Fig. 3. The flow is visualized for a single smooth wheel rotated at rates from 50 to $700 \mathrm{rpm}$, which corresponds to Reynolds number range from 3000 to 40000 . This operating range is representative for a city car driving cycle.

Figure 3 shows that the flow is divided into two parts, below and above the oil surface. In the upper part, the flow is governed by the interplay between the rotational inertia, gravity, surface tension, and viscous shear forces. In the below part the same forces except the surface tension are of importance. At low rotation rates $(50 \mathrm{rpm})$ the flow is laminar in both of these parts and the two fluid phases, oil and air, are distinctly separated. The flow problem in this case can be compared to a laminar rotating drum problem [11]. As rotational rate increases, the volume flow of the oil elevated by the wheel increases, and the streams of the oil flowing down from the wheel are also becoming stronger. The oil jet impinging into the oil pool results in air entrainment into the oil in the form of a void. With increased jet momentum, the void grows in size until it becomes unstable and collapses, and the mixing of the two fluids occurs with the formation of bubbles in the oil. The physical mechanism of air entrainment by a liquid jet is described in detail by Soh et al. [12].

The jet entrainment can either be laminar or turbulent, as our PIV data show, and the particular case depends on the flow Reynolds number. The laminar jet is undergoing the laminar-turbulent transition after the entrainment into the oil pool. In either case the entraining jet is creating the turbulence in the oil pool. As also shown by PIV measurements at $100 \mathrm{rpm}(R e=6000)$ the laminar-turbulent transition of the boundary layer on the submerged wheel surface occurs.

Due to further increase of the oil stream elevation with increased rotation rate $(150 \mathrm{rpm})$, the stream length increases and the pinch-off of the oil jet occurs, which caused by the Rayleigh-Plateau instability. At rotation rate of $150 \mathrm{rpm}$ the inertia and shear forces become large enough to transport the oil beyond the topmost wheel point which leads to the formation of a secondary oil stream on 

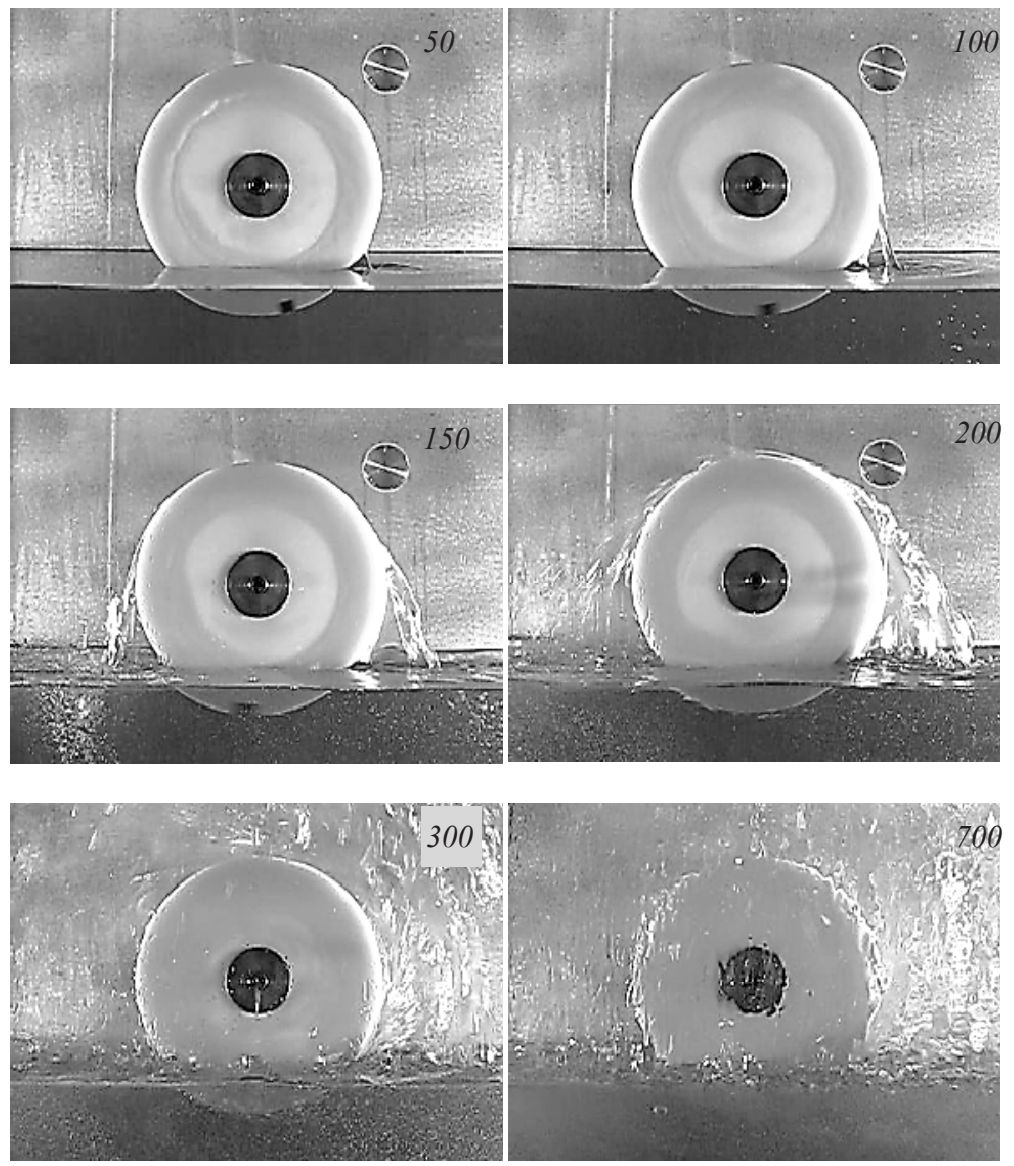

Figure 3: Smoothwheel at rotation rates of50, 100, 150, 200, 300, $700 \mathrm{rpm}$.Rotation direction is counter-clockwise, $R e=3000-40000$.

the left side of the wheel. This stream has larger velocity as compared to the primary stream since the wheel tangential velocity vector on this side is directed downwards, so the inertial and gravity forces acting in same direction downwards and accelerating the stream. At $150 \mathrm{rpm}$ rotation the secondary stream is having a larger momentum as compared to the primary stream and causes a deeper stream entrainment into the pool which in turn leads to the formation of a larger amount of air bubbles to the left of the wheel. At rotation rate of $200 \mathrm{rpm}$ the oil film leaves the wheel surface on the topmost point of the wheel. At this rotation rate the oil film thickness increases and the inertial force overcomes the viscous forces. At $300 \mathrm{rpm}$ the oil splashes are becoming strong enough to reach the top and side walls of the surrounding box. At $700 \mathrm{rpm}$ the oil splashing flow is so strong that the oil from the top wall is running in all directions and the oil streams can be observed on the front window of the box. These streams are responsible for a significant optical distortion seen on picture. 


\subsection{Air bubble visualizations}

The air bubbles in the oil pool are primarily generated by the entraining oil jets. The process of bubble creation was discussed in previous section. In current section the bubble distributions are considered.

In Fig. 4 raw PIV images are presented which are showing the bubbles around the wheel at different rotation rates. Each of visualizations is composed of two images which were taken at different experimental runs, i.e. not simultaneously.

The bubbles are uniformly lighted up by the fluorescent light emitted from the seeding particles and due to this appear as contours. This method of bubble visualization is commonly called as planar fluorescence for bubble imaging (PFBI) and the main advantage of PFBI that it can be applied in flows with large void fractions of bubbles, up to 5-6\% [13].

As seen in Fig. 4the air bubbles of various sizes are generated in the oil. The bubble generation starts form rotation rates of $100 \mathrm{rpm}$, and the concentration of bubbles increases with increased rotation rate. In Fig. 4 one can observe a variety of bubble shapes from nearly spherical to elongated, aerofoil-like shapes. The elongated bubbles are visible near the vicinity of the wheel where the counter action of the inertial/shear forces and the buoyancy acting on bubbles leads to their stretching.

The bubble occupation regions are also varying with the rotation rate. At 200 rpm the splash and bubble creation occur primarily near the wheel centre plane and the bubble layer is localized around this plane. The bubble occupation zone is limited to the region near the wheel. At higher rotation rates the splash streams are becoming more spread so the bubble generation zone increases and the bubble occupation zones also extend significantly. The oil motion leads to further distribution of the bubbles so they are occupying the entire pool. After a longer time interval the large bubble void fraction in the oil pool created far too large obstruction of the light path so the image quality decreased drastically. To overcome this effect, for rotation speeds of 400 and $1200 \mathrm{rpm}$ the measurements were performed only during a short time interval after the transient start of the wheel.

At $400 \mathrm{rpm}$ one can observe big bubbles which are rising up on the sides of the wheel. As one can expect these bubbles are not following the wheel rotation but inducing the vertical velocity and turbulence in the fluid [14] and acting as obstacles for the sidewall boundary layers on the wheel. The lifespan of the biggest bubbles is of the order of seconds and the smallest bubbles are remaining in the pool for significantly longer time, of order of 1 hour. As one can expect the smallest bubbles are following the flow streamlines very well.

For each rotation rate there is an own equilibrium state between the rate of bubble generation and the rate of bubble escape from the bath. Increased rate of the bubble generation leads to a larger bubble void fraction in the pool which in turn increases the rate of bubble merging and escaping. Of great interest is to study this process in detail which is planned in our future work. 

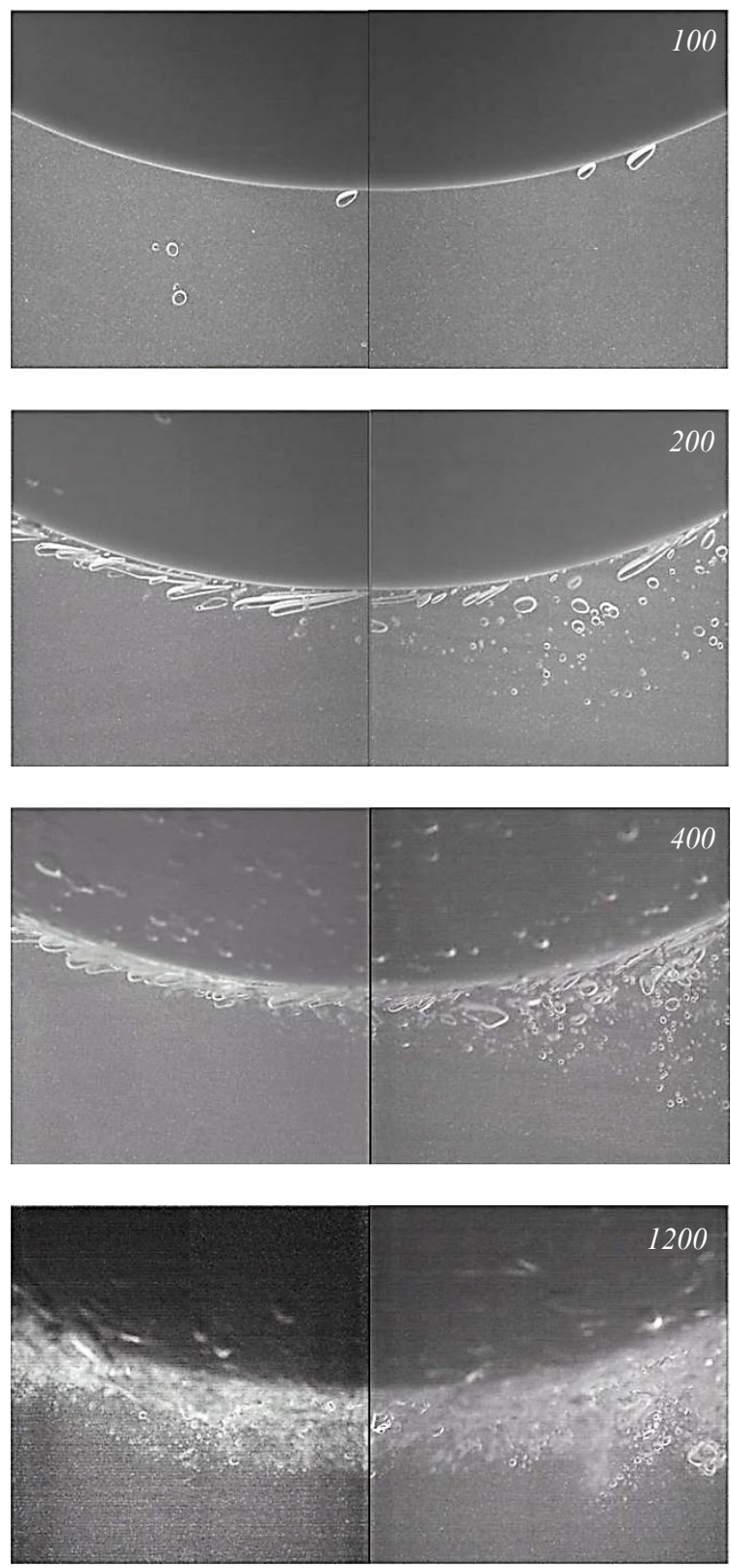

Figure 4: Air bubbles around the flat wheel at rotation rates 100, 200, 400, $1200 \mathrm{rpm}$. Wheel rotation direction is counter-clockwise. Note the elongated bubble shapes close to the wheel, and increase of bubble concentration at increased rotation rates. 


\subsection{Velocity fields}

The mean velocity fields around the wheel obtained at different rotation rates are presented in Fig. 5. One should notice that in this figure the velocity of the flow in the upper part (above the oil surface) has to be considered only as a qualitative result. Due to the fact that in the region above the oil surface the PIV technique is applied to the oil drops instead of the seeding particles, and presence of strong non-uniformities of illumination intensity and multiple light scattering, the reliability of the data is poor. From other hand the top part of the flow is of minor interest since the motion of the oil droplets is relatively simple and described by ballistic parabolic trajectories.

From Fig. 5 it is clearly seen that as the rotation rate increases the viscositydominated flow is changed to the flow with dominated inertial forces. As a consequence, the characteristic scales of the flow change. For the bottom part the characteristic scale is the thickness of the boundary layer. The thickness of the boundary layer decreases with increased inertial forces. For the top part the characteristic scale is the flying distance of the oil droplets. The flying distance increases with increased inertial forces since the initial velocity of the oil drops leaving the surface of the wheel increases.
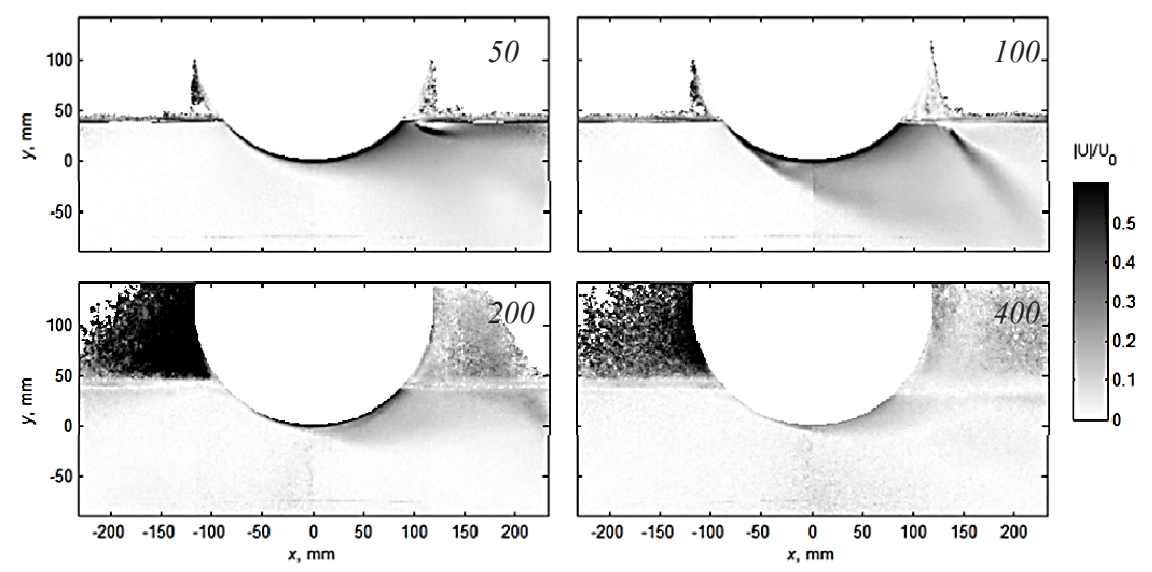

Figure 5: Contours of average velocity magnitude at rotation rates of 50, 100, 200 , and $400 \mathrm{rpm}$.

Analysis of the boundary layer profiles on the wheel surface has revealed that the boundary layer is laminar at $50 \mathrm{rpm}$, transitional at $100 \mathrm{rpm}$ and turbulent at rotation rates above $200 \mathrm{rpm}$.

The breakdown of the oil jet which is emitted from the surface of the wheel and enters the pool is illustrated in Fig. 6 for case of $100 \mathrm{rpm}$ rotation rate. One can observe that the initially laminar jet undergoes the instability phase and become turbulent. At higher rotation rates the oil jets entering the pool become turbulent nearly immediately after the entrance. 


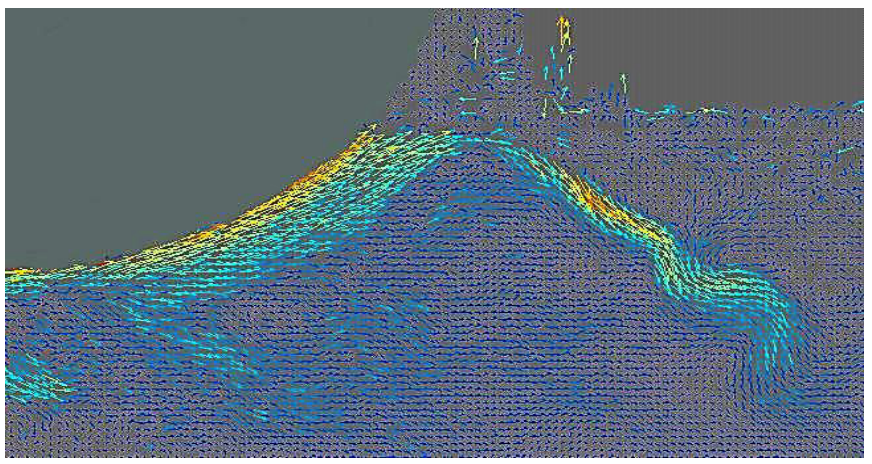

Figure 6: Instantaneous velocity vector field showing the oil jet entrainment and breakdown. $100 \mathrm{rpm}$ case.

In fact for successful description of the multiphase flow the phases involved have to be discriminated. Ideally for each phase the velocity and the void fraction have to be determined. Among the methods which are commonly used for the discrimination of the bubble phase, see [13-19], none is offering a good enough reliability of the bubble recognition in the case of significantly irregular bubble shapes and high degree of mutual bubble overlap. This means that in our case the bubble discrimination appeared to be a non-trivial task. From other hand the fluid phase discrimination was performed successfully by using common PIV processing methods. The processing steps involve use of very strict settings for the peak ratio criteria (peak ratio 2) and for the median filter criteria (1 r.m.s. of the neighbours). As a result the most of the slow bubbles and bubble shadowed zones were removed from the velocity fields. The result was further improved by applying a3 $\sigma$ threshold to the time-series of images and removing the vectors having far too small or excessive amplitudes as compared to other vectors in the same time-series. A typical result after the processing is illustrated in Fig. 7. The figure shows that the largest and slowest bubbles as well as the bubble shadows are successfully removed from the velocity field. The smallest bubbles follow the oil flow very well and are preserved.

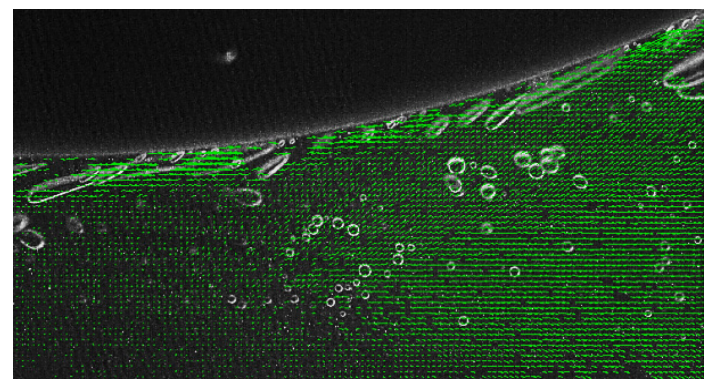

Figure 7: PIV vector field combined with a raw image demonstrating that the slow-moving bubbles and the bubble shadows are not contributing to the velocity field. Every second vector is shown; case of $200 \mathrm{rpm}$ rotation rate. 


\subsection{Comparison with spur gear wheel}

Flow around a spur gear wheel has its own peculiarities. The air is trapped between the teeth of the wheel, see Fig. 8. The volume of the trapped air increases with increased rotation rate. Also, the splash strength increases significantly since larger amount of oil is dragged in the teeth pockets. From other hand, for the submerged part of the wheel the fluid in the pockets between the teeth is moving with same velocity as the wheel itself. Due to teeth the boundary layer developed on the wheel surface is undergoing transition to turbulence quicker. The scaling of the boundary layer thickness is shown in Fig. 9 in comparison to smooth wheel.
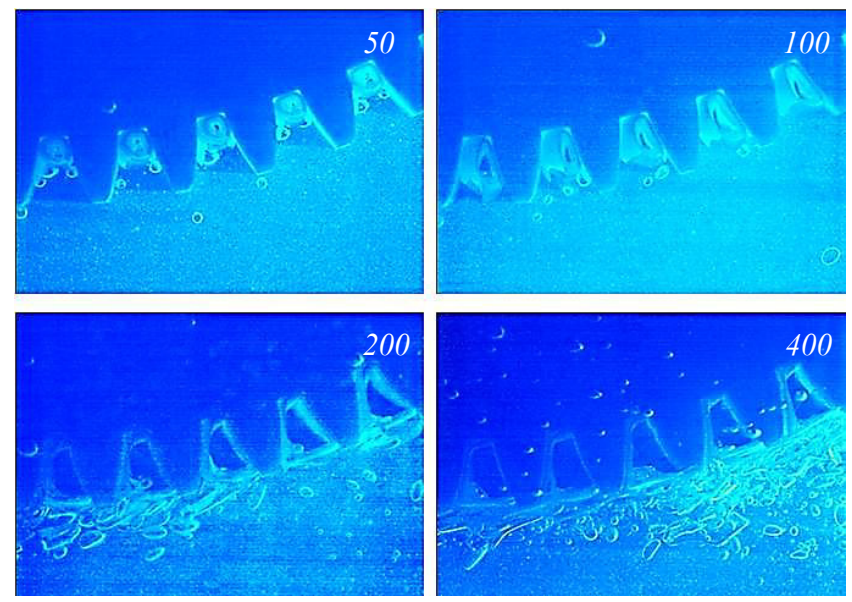

Figure 8: Air bubbles betweenthe teeth and in the vicinity of a spur gearwheel at rotation rates 50, 100, 200, and $400 \mathrm{rpm}$.

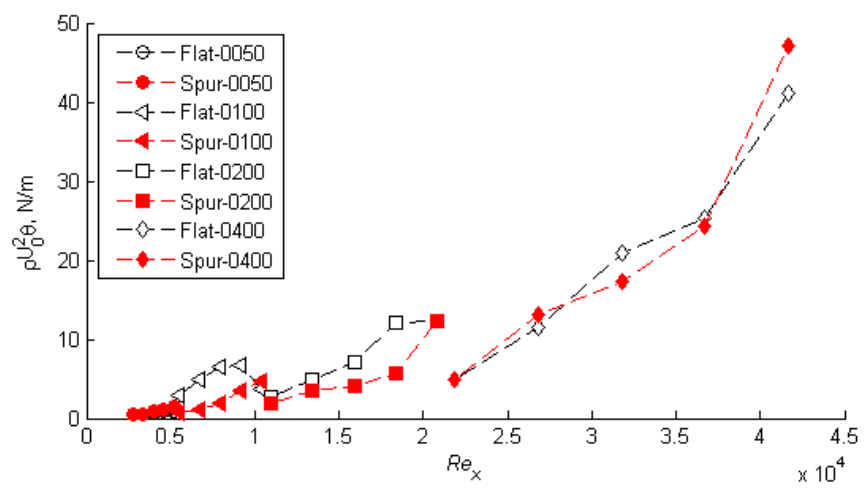

Figure 9: Boundary layer momentum thickness for flat and spur gear wheelsshown in normalized coordinates. 


\section{Conclusions}

Flow around a single rotating wheel within a modelled gearbox is investigated by means of visualizations and PIV. Two-component PIV velocity measurements were successfully performed for several different rotation rates of the wheel. The results of study demonstrate that the flow around the wheel is very complex. The rotation of the wheel results in elevation of the oil by the wheel and creation of the streams of the oil ejecting from the wheel, i.e. the oil splash. The streams can break-up and lead to formation of oil drops. With increased rotation rates the splashed oil streams start to agitate the surface of the oil pool and collide with the gearbox walls. The oil streams entering the oil pool lead to generation of air bubbles in the oil. The penetration of the oil jets into the oil bath and motion of the air bubbles are creating turbulence in the oil.

The splash increases with increased rotation rate due to increased role of inertial forces. From other hand, for the flow inside the pool the prevalence of the inertial forces over the viscous forces leads to thinner boundary layers and more and more confined regions of induced flow very close to the wheel.

\section{Acknowledgements}

Authors would like to thank SAAB Powertrain, Sweden for providing the experimental rig and financial support for this project. Many thanks to Ehsan Sistani for taking part in the experiments.

\section{References}

[1] Honkanen M., Eloranta H., and Saarenrinne P. Digital imaging measurement of dense multiphase flows in industrial processes. Flow Meas. Instrum. 21, pp. 25-32, 2009.

[2] Petry-Johnson T.T., Kahraman A., Anderson N.E., Chase D.R. An experimental investigation of spur gear efficiency, ASME J. Mech. Des. 130, 062601, 2008.

[3] Changenet C., and Velex P., A model for the prediction of churning losses in geared transmissions-preliminary results, ASME J. Mech. Des. 129, pp. 128-133, 2007.

[4] Eastwick C.N., Johnson G. Gear windage: a review, ASME J. Mech. Des. 130, 034001, 2008.

[5] Höhn B.-R., Michaelis K. and Hinterstoißer M. Optimization of gearbox efficiency. Goriva i maziva 48 (4) 441-480, 2009.

[6] Al-Shibl K., Simmons K., and Eastwick C.N. Modelling windage power loss from an enclosed spur gear. Proc. I Mech E, Vol. 221, Part A: J. Power and Energy, 2007.

[7] Hill M.J., Kunz R.F., Noack R.W. Long L.N., Morris P.J., Handschuhk R.F. Application and validation of unstructured overset CFD technology for rotorcraft gearbox windage aerodynamics simulation. In proc. of American Helicopter Society 64th Annual Forum, Montreal, Canada, 2008. 
[8] Lemfeld F., Fraňa K., Unger J., Numerical simulations of unsteady oil flows in the gear-boxes, J. Applied Science in the Thermodynamics and Fluid Mechanics 1, pp. 27-32, 2008.

[9] Moshammer T., Mayr F., Kargl K., and Honeger C. Simulation of oil flow in gear box housing, SAE Int., 2006-01-1574, 2006.

[10] Li L., Versteeg H.K., Hargrave G.K., Potter T., and Halse C. Numerical investigation on fluid flow of gear lubrication, SAE Int., 2008-01-1650, 2008.

[11] Hasan N., Naser J. Determining the thickness of liquid film in laminar condition on a rotating drum surface using CFD. Chem. Eng. Sci. 64, pp. 919-924, 2009.

[12] Soh W.K., Khoo B.C., Yuen W.Y.D. The entrainment of air by water jet impinging on a free surface, Exp. Fluids 39, pp. 496-504, 2005.

[13] Akmetbekov Y.K., Alekseenko S.V., Dulin V.M., Markovich D.M., Pervunin K.S. Planar fluorescence for round bubble imaging and its application for the study of an axisymmertric two-phase jet. Exp. Fluids 48, pp. 615-629, 2010.

[14] Riboux G., Risso F., Legendre D. Experimental characterization of the agitation generated by bubbles rising at high Reynolds number, J. Fluid Mech. 643, pp. 509-539, 2010.

[15] Sridhar G., Ran B., Katz J. Implementation of PIV to multiphase flow. Cavitation and Multiphase Flow Forum ASME-FED-Vol.109, pp. 205210, 1991.

[16] Dehaeck S., van Beeck J.P.A.J., Riethmuller M.I. Extended glare points velocimetry and sizing for bubbly flows. Exp. Fluids 39, pp. 407-419, 2005.

[17] Hassan Y.A, Blanchat T.K., Seeley Jr. C.H., Canaan R.E. Simultaneous velocity measurements of both components of a two-phase flow using particle image velocimetry. Int. J. Multiphase Flow 18, pp. 371-395,1992.

[18] Honkanen M. Turbulent multiphase flow measurements with digital particle image velocimetry: Application to bubbly flows. MSc thesis, Tampere University of Technology, 2002.

[19] Lindken R. and Merzkirch W. A novel PIV technique for measurement in multiphase flows and its application to bubbly flows. Exp. Fluids 33, pp. 814-825, 2002. 